\title{
Spectro-temporal neural dynamics during sentence completion
}

4 Tim Coolen ${ }^{\mathrm{a}, \mathrm{b},{ }^{*}, \text { Alexandru Mihai Dumitrescu }}{ }^{\mathrm{a},{ }^{*},}$, Vincent Wens ${ }^{\mathrm{a}, \mathrm{c}}$, Mathieu

5 Bourguignon ${ }^{\mathrm{a}, \mathrm{d}, \mathrm{e}}$, Gustavo Lucena Gómez ${ }^{\mathrm{a}}$, Antonin Rovaia ${ }^{\mathrm{a}}$, Niloufar Sadeghib, Charline

6 Urbain $^{\mathrm{a}, \mathrm{f}}$, Serge Goldman ${ }^{\mathrm{a}, \mathrm{c}}$, Xavier De Tiège ${ }^{\mathrm{a}, \mathrm{c}}$

aLaboratoire de Cartographie fonctionnelle du Cerveau, ULB Neuroscience Institute (UNI), Université libre de Bruxelles (ULB), Brussels, Belgium.

11 bDepartment of Radiology, CUB - Hôpital Erasme, Université libre de Bruxelles (ULB),

12 Brussels, Belgium.

13 Magnetoencenphalography unit, Clinics of Functional Neuroimaging, Service of Nuclear

14 Medicine, CUB - Hôpital Erasme, Université libre de Bruxelles (ULB), Brussels, Belgium.

15 dBCBL - Basque Center on Cognition, Brain and Language, 20009 San Sebastian, Spain.

eLaboratory of Neurophysiology and Movement Biomechanics, ULB Neuroscience

17 Institute (UNI), Université libre de Bruxelles (ULB), Brussels, Belgium.

18 fUR2NF, Neuropsychology and Functional Neuroimaging Research Unit, Centre for Research in Cognition and Neurosciences (CRCN), ULB Neuroscience Institute (UNI),

20 Université Libre de Bruxelles(ULB), Brussels, Belgium.

$21 *$ Equal contribution.

24 Corresponding author: Tim Coolen, Department of Radiology, CUB - Hôpital Erasme,

25 Université libre de Bruxelles (ULB), 808 route de Lennik, Brussels, Belgium. E-mail:

26 tim.coolen@ulb.be Tel: +32.2.555.47.11. 


\section{Abstract}

This magnetoencephalography (MEG) study aimed at characterizing the spectrotemporal dynamics of brain oscillatory activity elicited by sentence completion (SC). For that purpose, we adapted a version of the SC experimental paradigm typically used in functional magnetic resonance imaging to MEG investigation constraints. Twenty righthanded healthy young adults underwent MEG recordings while they were sequentially presented with short sentences divided in three parts: the first two giving context and the last requiring completion. MEG data were then analysed using a prior-free, nonparametric statistical approach with stringent control of the family-wise error rate. We identified three successive significant neural response patterns associated with distinct spatial and spectro-temporal characteristics: (i) an early ( $<300 \mathrm{~ms}$ ) bioccipital 4-10-Hz event-related synchronization (ERS); (ii) an intermediate (at about $400 \mathrm{~ms}$ ) 8-30-Hz event-related desynchronization (ERD) in an extended semantic network involving the ventral language stream as well as bilateral posterior nodes of the default mode network (DMN) in both hemispheres; (iii) a late (>800 ms) 8-30 Hz ERD involving the left dorsal language stream. Furthermore, the left component of the ventral language stream displayed prolonged ERD after $800 \mathrm{~ms}$ compared to the right which showed signs of inhibition in the form of ERS. Overall, this study elucidates the dynamics of the recruitment of the language network that accompany SC and the spectro-temporal signature of an extended semantic network. This MEG adaptation of an SC paradigm also paves the way for novel approaches in presurgical language mapping and may help to understand the neural underpinnings of the alterations of sentence completion in various neurologic 50 disorders affecting language.

53 Keywords: Sentence completion, Semantic Network, Ventral Stream,

54 Magnetoencephalography, Oscillations 


\section{Introduction}

Sentence completion (SC) is a well-documented language task in the functional magnetic resonance imaging (fMRI) literature (e.g., Ashtari et al., 2005; Barnett et al., 2014; Black et al., 2017; Kircher et al., 2001; Petrella et al., 2006; Salek et al., 2017; Wilson et al., 2017; Zaca et al., 2012; Zacà et al., 2013). It is endorsed by the American Society of Functional Neuroradiology as a first-choice experimental paradigm for language functional brain mapping (Black et al., 2017) that is typically constructed as a block design (for a description of fMRI designs, see, e.g, Amaro \& Barker, 2006). In such design, the active condition usually consists of short written sentences with a missing final part that participants are asked to complete, while the control condition includes low-level visual stimuli such as gibberish written sentences with a missing final part. Likely due to the high linguistic complexity, inherent to sentence-level processing (Vigneau et al., 2006), SC robustly (Black et al., 2017; Zacà et al., 2013) and reliably elicits increases in blood oxygen level dependent (BOLD) signal in the left perisylvian regions (Wilson et al., 2017) deemed essential to language function (for a review, see, e.g., Tremblay \& Dick, 2016).

Owing to its exquisite temporal resolution, magnetoencephalography (MEG) provides the opportunity to investigate the spectral and temporal oscillatory neural dynamics underlying sentence processing within left-lateralized language-related regions. Previous MEG studies (e.g., Halgren et al., 2002; Hultén et al., 2019; Kielar et al., 2015; Lam et al., 2016; Meltzer et al., 2017; Meltzer \& Braun, 2011; Piai et al., 2015; Wang et al., 2018) did not investigate the neural oscillatory dynamics that accompany the completion of sentences during SC. Instead, they focused on the semantic integration of incoming words in the sentence context (Halgren et al., 2002; Hultén et al., 2019; Lam et al., 2016; Wang et al., 2018). Other studies investigated the effect of the modulation of the phonological and semantic aspects of the sentence (Meltzer et al., 2017) or of specific linguistic abnormalities such as semantic violations (Kielar et al., 2015). Moreover, the

83 sentence endings were given in those studies, or cued by a picture in one of them (Piai 84 et al., 2015) which focused on production aspects. 
This MEG study was therefore performed to characterize the spectral, temporal and spatial dynamics of the neural events associated with the whole SC process. For that purpose, we developed a version of the classic fMRI SC experimental paradigm adapted to the constraints of MEG investigations (for a detailed description, see, e.g., Gross et al., 2013). We first analyzed the whole-brain MEG data acquired in right-handed healthy young adults using a prior-free, non-parametric statistical approach with stringent control of the family-wise error rate. Secondly, we restricted the investigation to the bilateral

92 ventral stream of language (Hickok \& Poeppel, 2007) to shed light on the relative 93 contribution of its left and right components to sentence processing. Indeed, in addition

94 to the typically left-sided changes elicited by sentence stimuli in left-sided language95 related areas (Tzourio-Mazoyer et al., 2017), both fMRI (e.g., Barnett et al., 2014; Wilson et al., 2017; Zaca et al., 2012) and MEG (e.g., Halgren et al., 2002; Hultén et al., 2019; Kielar et al., 2015; Lam et al., 2016; Meltzer et al., 2017) studies also reported contralateral homologous right-sided activity modulations, notably in right posterior temporal regions (e.g., Kircher et al., 2001; Meltzer et al., 2017). Still, the functional role of these right-sided temporal changes during sentence processing remains poorly understood. They may correspond to context processing (Vigneau et al., 2010) or prolonged maintenance of multiple semantic representations necessary to understand the gist of the sentence (Kircher et al., 2001). We expected that, due its exquisite temporal resolution, MEG will yield novel insights into the different functional roles of left and right components of the ventral language stream through potentially divergent spectrotemporal fingerprints. 


\section{Materials and methods}

108

109

\subsection{Subjects}

Twenty right-handed healthy adult subjects (mean age: $31.2 \pm 8.1$ years, range: 22.0 - 51.4 years; 11 females) were included in this study. None of them had a prior history of neurologic, psychiatric or learning disorder, nor MRI contraindication. All subjects were right-handed $(93.3 \pm 8.8 \%$; range: $77.8-100 \%)$ according to the Edinburgh Handedness Inventory scale (Oldfield, 1971).

All subjects contributed to the study after giving written informed consent. They were given a small financial incentive for their participation. The study received prior approval by the Ethics Committee of the CUB - Hôpital Erasme (Université libre de Bruxelles, Brussels, Belgium; REF: P2017/272)

\subsection{SC paradigm}

A total of 72 simple short sentences in French were created (listed in Supplementary Table 1). Each sentence was divided into three parts. The first part (P1) contained the subject, composed of a common noun and its determiner (mean of $2.04 \pm$ 0.31 words; $10.47 \pm 2.59$ characters, including spaces; e.g., "The hen"). The predicate was partly stated in the second part (P2; $2.53 \pm 0.73$ words; $12.36 \pm 3.29$ characters; e.g., "lays an") and had to be completed by the participants in the third part (P3), which prompted the completion by a visual clue (" . .).

A schematic representation of the paradigm is provided in figure 1. A block structure, typical for a clinical fMRI SC paradigm, served as the canvas for this MEG adaptation and consisted of six "task" blocks of $60 \mathrm{~s}$ each, alternating with "rest" periods of $30 \mathrm{~s}$. Total paradigm duration was $9.6 \mathrm{~min}$. The task blocks of interest contained six sentences. A fixation cross lasting $1 \mathrm{~s}$ preceded every sentence, and each part thereof (i.e., P1, P2, P3) was shown for $3 \mathrm{~s}$. The thirty-six sentences were different and randomly picked among the available sentence database for each participant. Rest periods consisted of three sequences that were created to visually resemble the target sentences and also divided in a first and a second part, composed only of "*" characters and spaces, 
137 followed by the same empty third part (" .). For added clarity, a $1 \mathrm{~s}$ cue 138 followed by a $2 \mathrm{~s}$ fixation cross announced the beginning of each block.

Subjects were instructed to silently read the first two parts of the sentences and to

140 silently generate the sentence endings with one or few words when presented with the 141 P3 completion cue. During the rest periods, they were asked to simply look at the screen.

142 Prior to the MEG recording, participants were trained on a separate set of six sentences 143 and asked to overtly read and complete the sentences to ensure they correctly 144 understood the instructions. All subjects understood the task and performed the training 145 correctly.

146 Visual stimuli appeared in white Times New Roman font on a black background.

147 They were projected onto a screen placed at the feet of the MEG bed and made visible 148 to the subject through an oblique mirror so that the visual angle did not exceed $7^{\circ}$. The 149 appearance of each sentence part (P1, P2 and P3) was marked by a specific trigger 150 signal recorded in a separate channel synchronously to the MEG data. 
A) Paradigm block structure (6 repetitions)

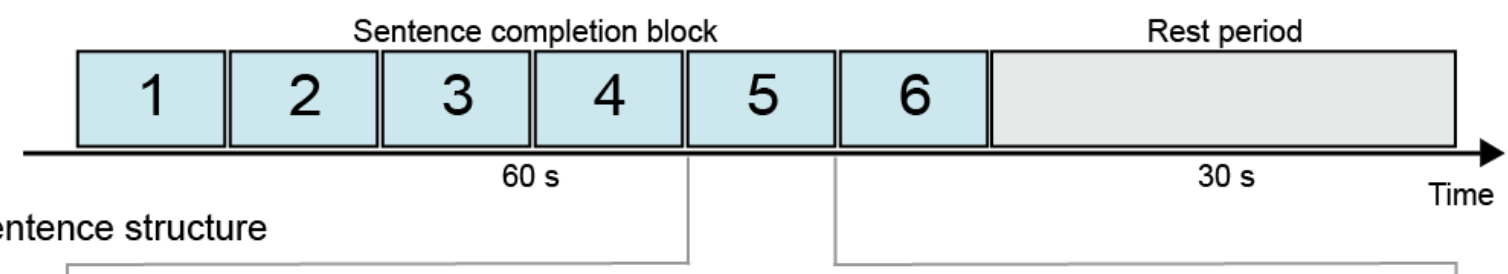

B) Sentence structure

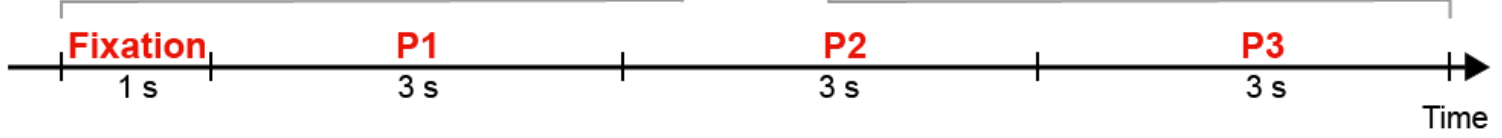

C)

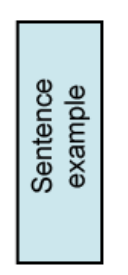

\section{Fixation}

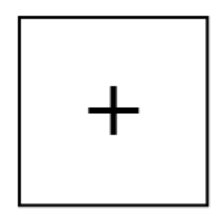

\section{P1}

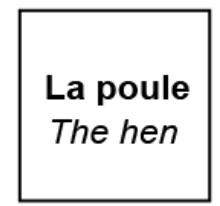

P2

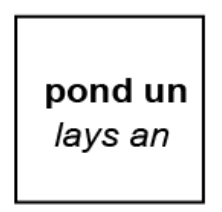

P3

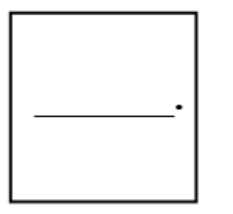

153 Figure 1: Schematic representation of the SC paradigm. A) The general block

154 structure consisted of task blocks of six sentences (blue), alternating with rest periods 155 (grey). B) The sentence structure was composed of a fixation cross followed by a first 156 (P1: determiner and noun), second (P2: part of predicate) and third (P3: to complete) part. 157 C) Example sentence. The text in French actually presented to the subjects is written in 158 bold. For convenience, the English translation is also provided here in italic. 


\subsection{Data acquisition}

MEG data were recorded (band-pass: $0.1-330 \mathrm{~Hz}$; sampling rate: $1 \mathrm{kHz}$ ) in a lightweight magnetically shielded room (Maxshield ${ }^{\mathrm{TM}}$, MEGIN, Croton Healthcare, Helsinki, Finland; see De Tiège et al., 2008 for more details) using a 306-channel wholescalp-covering neuromagnetometer (Triux ${ }^{\mathrm{TM}}$, MEGIN, Croton Healthcare, Helsinki, Finland). Subjects were installed in the supine position to minimize head movement artifacts.

Four fronto-mastoid head-tracking coils monitored the subjects' head position inside the MEG helmet. The locations of the coils and at least 350 head-surface points (on scalp, nose and face) with respect to anatomical fiducials were recorded with an electromagnetic tracker (Fastrak, Polhemus, Colchester, VT, USA) before starting the MEG session.

For MEG source localization, an anatomical 3D T1-weighted gradient echo sequence $\left(\right.$ time of repetition $=8.2 \mathrm{~ms}$, time of echo $=3.1 \mathrm{~ms}$, flip angle $=12^{\circ}$, field of view $=24 \mathrm{~cm}$, matrix $=240 \times 240$, isotropic $1 \mathrm{~mm}^{3}$ voxels) of the head was also acquired for each subject using a hybrid 3T SIGNA TM PET-MR scanner (GE Healthcare, Milwaukee, Wisconsin, USA) with a 24-channel head and neck coil.

\subsection{MEG data preprocessing}

Raw MEG data were preprocessed off-line using signal space separation (Taulu et al., 2005) to reduce external interferences and correct for head movements (MaxFilter ${ }^{\mathrm{TM}}$ v2.2 with default parameters, MEGIN, Croton Healthcare, Helsinki, Finland). Cardiac, eye-movement and electronic artifacts were then identified by independent component analysis (FastICA algorithm with rank reduction to 30 and nonlinearity tanh; Hyvärinen \& Oja, 2000; RRID: SCR_013110) applied to sensor time series filtered between 0.5 and $45 \mathrm{~Hz}$ (Vigario et al., 2000) and visual inspection of the components (mean number of artifactual components per subject : $2.30 \pm 0.57$ ). Artifactual components were then regressed out from the full-rank data.

The preprocessed MEG data were further handled with Fieldtrip (Oostenveld et al., 2011; RRID:SCR_004849). The continuous data were first segmented in 6 s-long epochs covering each sentence part P1, P2 or P3 (2000 ms prestimulus to 4000 ms post- 
stimulus, each stimulus time being identified by a trigger event). Epochs with absolute signal values exceeding $3 \mathrm{pT}$ in at least one magnetometer or $0.7 \mathrm{pT} / \mathrm{cm}$ in at least one gradiometer were discarded as likely contaminated by artifacts.

The time-frequency Fourier coefficients were then estimated using a 7-cycle Morlet wavelet decomposition, for each of the 306 MEG channels (102 magnetometers and 204 planar gradiometers). This was done in a window of interest starting at 500 ms before and ending at $3000 \mathrm{~ms}$ after each sentence part (P1, P2, P3) with a 50 ms time increment. Of note, the usage of wider (6 s-long) epochs was necessary to probe the lowest frequencies in this window of interest. The Fourier coefficients were estimated from $1 \mathrm{~Hz}$ to $45 \mathrm{~Hz}$ by steps of $1 \mathrm{~Hz}$.

\subsection{Source reconstruction}

Individual anatomical MRIs were segmented using the Freesurfer software (Fischl, 2012; Martinos Center for Biomedical Imaging, Massachusetts, USA; RRID: SCR_001847). MEG and MRI coordinate systems were co-registered using the three anatomical fiducial points for initial estimation and the head-surface points to manually refine the surface co-registration.

Individual MEG forward models were then computed using the single-layer Boundary Element Method implemented in the MNE-C software suite (Gramfort et al., 2014; Martinos Center for Biomedical Imaging, Massachusetts, USA; RRID: SCR_005972). To ease the group-level analysis, forward models were based on a source grid obtained from a common 5-mm cubic grid (containing 16102 source locations), built in the Montreal Neurological Institute (MNI) template brain by applying the non-linear spatial deformation estimated with the algorithm implemented in Statistical Parametric Mapping (SPM12, Wellcome Department of Cognitive Neurology, London, UK; RRID: SCR_007037). Three orthogonal current dipoles were placed at each grid point.

The resulting forward models were then inverted via Minimum Norm Estimation (MNE; Dale \& Sereno, 1993). The sensor-space noise covariance was estimated from 5 minutes of artifact-free data recorded from an empty room preprocessed using signal space separation and filtered between 0.5 and $45 \mathrm{~Hz}$. The regularization parameter was derived for each condition (P1, P2 and P3) from the signal-to-noise level estimated from 
221 the noise covariance and the covariance of the concatenated epoch data (Wens et al., 222 2015). The depth bias was corrected by noise standardization (Pascual-Marqui, 2002).

223 The MNE inverse operator was then used to project the sensor-level time-frequency 224 Fourier coefficients at each epoch and obtain the time-frequency Fourier coefficients of 225 each dipole component of the 16102 sources. The amplitude of each source was finally 226 obtained as the Euclidean norm of the magnitude of the three corresponding fourier 227 coefficients and averaged over epochs. This led to one source distribution of time228 frequency power map per condition (P1, P2 and P3) and subject, which was then used 229 for subsequent analysis.

\subsection{Event-related (de)synchronization measures}

A baseline was defined as the period of time preceding the onset of the first part of the sentence (P1), from $500 \mathrm{~ms}$ to $100 \mathrm{~ms}$ prestimulus, i.e., during the display of the fixation cross. Event-related "enhancement" or "synchronization" (ERS) was defined as post-stimulus power increase in a given frequency band compared to the baseline (Pfurtscheller, 2001). Conversely, event-related "suppression" or "desynchronization" (ERD) was defined as a power decrease (Pfurtscheller, 2001). This baseline was chosen to measure neural processes unfolding as the sentence progressed, including sustained language-related activity, in distinction, e.g., with Hultén et al. (2019) who focused on the effect of the previous context on upcoming stimuli.

In practice, source-level ERS/ERD measures were obtained for each frequency bin by dividing its value with the mean baseline value at the same frequency and 243 subtracting 1.

\subsection{Statistical analyses}

246 Group-level statistical analysis of the trial-averaged data was performed with a 247 maximum $t$ statistic procedure (Blair \& Karniski, 1993), which provides a rigorous control 248 of the family-wise error (FWE) rate in the context of multidimensional MEG data (16102 249 sources $\times 45$ frequency bins $\times 60$ time points), fraught with correlations among 250 neighbouring measurements, while limiting the loss of statistical power (Groppe et al., 251 2011). For each sentence part (P1, P2 and P3), we started by computing a one-sample $t$ 
statistic for each source-time-frequency point of our dataset. This corresponds to a massunivariate test against the null hypothesis that conditions induce no power changes compared to the baseline, i.e., no ERS or ERD. The maximum absolute $t$ value across this dataset defined the maximum statistic. Its null distribution was generated nonparametrically by recomputing 2000 times a similar statistic after changing the sign of the relative amplitude change data in a random selection of subjects. This amounts to the fact that ERS and ERD are exchangeable under the null. The 95th percentile of this null distribution of maximum was used to establish a significance threshold at a $95 \%$ confidence level, fully corrected. All supra-threshold values were deemed significant.

\subsection{Global spectro-temporal neural dynamics of SC}

In order to obtain an overview of the spectro-temporal neural dynamics of the three constituent parts of the SC paradigm, a global time-frequency analysis map was constructed as the mean $t$-value across all sources. Then, only the supra-threshold $t$ values were included to locate the time-frequency points disclosing significant ERS (positive values) or ERD (negative values).

2.9. Frequency band and time-resolved mapping associated with SC

The source location associated with supra-threshold power modulations were projected onto a standard MNI brain and visualized with Mricron (Rorden \& Brett, 2000; RRID:SCR_002403) to create a frequency and time-resolved functional mapping. This source-space analysis was based on the single statistical threshold obtained from the unique analysis considering all time (60) and frequency (45) points for each of the three sentence parts. Given that a large number of such maps may be built (60 time points $x$ 45 frequency bins), we considered several averages within sliding frequency bands and time windows, allowing for the exploration of ERS/ERD dynamics on different spectro-

278 temporal scales. We first investigated spectrally-resolved maps averaged over the whole post-stimulus period (0-3000 ms) and over classical frequency bands (theta: 4-7 Hz; alpha: 8-11 Hz; low-beta: 12-20 Hz; high-beta: 21-30 Hz; low-gamma: 31-45 Hz). Their temporal development was assessed by averaging over the whole frequency spectrum 
283 followed by a last longer window of $1000 \mathrm{~ms}$. This rather coarse temporal segmentation was chosen based on the global time-frequency graph (see Results).

A detailed list of statistically significant local maxima was created for the bandlimited maps for further analyses. These local maxima were identified on the thresholded maps further smoothed with an isotropic gaussian kernel (full width at half maximum $=8$ $\mathrm{mm}$ ). Local maxima were discarded if they did not fall within the cortical parcels of the Automated Anatomical Labelling atlas (AAL; Tzourio-Mazoyer et al., 2002; RRID: SCR_003550) and the cerebellar hemispheres to capture plausible cortical activity. time window, a laterality index (LI) was extracted from the corresponding maps as the relative difference between the number $L$ of supra-threshold sources in the left hemisphere and the number $\mathrm{R}$ of supra-threshold sources in the right hemisphere, i.e., $L I=(L-R) /(L+R)$. A positive $\mathrm{LI}$ thus indicates a leftward dominance, with the maximum value $\mathrm{LI}=1$ reached if significant sources occur only in the left hemisphere. Likewise, negative LI indicates rightward dominance and the minimum LI = -1 is reached if significant effects arise in the right hemisphere only. A separate LI was calculated for ERS and ERD.

2.10 Spectro-temporal neural dynamics in the right vs. left ventral language stream during SC

A region of interest $(\mathrm{ROI})$ covering the ventral language stream was constructed on the basis of a meta-analytic language map (association test map; $p<.01$ corrected for false discovery rate; 1101 studies included; methodological details available on http://neurosynth.org) obtained with the Neurosynth online tool (Yarkoni et al., 2011; RRID:SCR_006798). The map was smoothed with an isotropic Gaussian kernel (full width at half maximum $=10 \mathrm{~mm}$ ) to account for the difference in location between fMRI activations and MEG sources, which is of the order of the centimetre (e.g., Stippich et al., 2007). A subset of the smoothed and binarized (z-score>0) left-sided map belonging to the posterior middle/inferior temporal (Hickok \& Poeppel, 2007) and fusiform (Saur et al., 2008) AAL parcellations (Tzourio-Mazoyer et al., 2002; RRID: SCR_003550) with MNI $y$ coordinates between $-32 \mathrm{~mm}$ (posterior edge of Heschl's gyrus parcellation) and -76 mm 
314 (posterior edge of middle temporal gyrus) was defined as the left part of the ventral 315 stream. Its mirror image constituted the right-sided counterpart. The two ROIs are 316 represented in Figure 5 (rightmost column).

317 Time-frequency values spatially-averaged within ROI were used to probe neural 318 oscillatory modulations potentially hidden by the stringent statistical threshold imposed by 319 the whole-brain analysis (i.e., loss of sensitivity due to the use of maximum statistics and 320 the large amount of comparisons), and to perform pairwise comparisons of the right vs. 321 left ROls.

322 Statistical testing of this difference was based on a maximum statistics procedure 323 similar to the whole-brain analysis but spatially restricted to the ROI. Here, univariate $t$ 324 statistics corresponded to two-sample paired $t$-tests and the null distribution was derived 325 by randomly permuting the labels 'left' and 'right' among subjects before computing these 326 t-values and extracting their maximum across time, frequencies, and sources in the ROI 327 (number of permutations: 2000).

2.11. Data availability statement

The MEG data used in this study will be made available upon reasonable request 332 Erasme and Université libre de Bruxelles). 


\section{3}

334

335

336

337

338

339

340

341

342

343

344

345

346

347

348

349

\section{Results}

\subsection{Global spectro-temporal neural dynamics of SC}

The general pattern of the spectro-temporal neural dynamics of SC was visualized using whole-brain-averaged time-frequency maps of ERS/ERD (Figure 2). It consisted of an immediate post-stimulus ERS in the lower frequency range (mainly $<10 \mathrm{~Hz}$ ) at the onset of each sentence part, though only the initial ERS in P1 in the theta/alpha range (5$8 \mathrm{~Hz}$ ) reached significance for a short period of time $(0-300 \mathrm{~ms})$. This ERS was followed by an ERD peaking around $400 \mathrm{~ms}$ mainly in the alpha and beta frequency bands. Significant ERD took place from $250 \mathrm{~ms}$ to $600 \mathrm{~ms}$ in P1, between $350 \mathrm{~ms}$ and the rest of the post-stimulus period in P2 and essentially between $300 \mathrm{~ms}$ and $1800 \mathrm{~ms}$ in P3. The frequency range of significant ERD was more limited in $\mathrm{P} 1$ (alpha and high beta bands) while it stretched from theta to low gamma bands in P2 and P3.

Given the temporal spread of the significant ERD in P2 and P3, five nonoverlapping $400 \mathrm{~ms}$ time windows were selected to cover the first $2 \mathrm{~s}$ of the post-stimulus period, followed by a final longer 1000 ms window to study the last second that was only relevant in $\mathrm{P} 2$. 

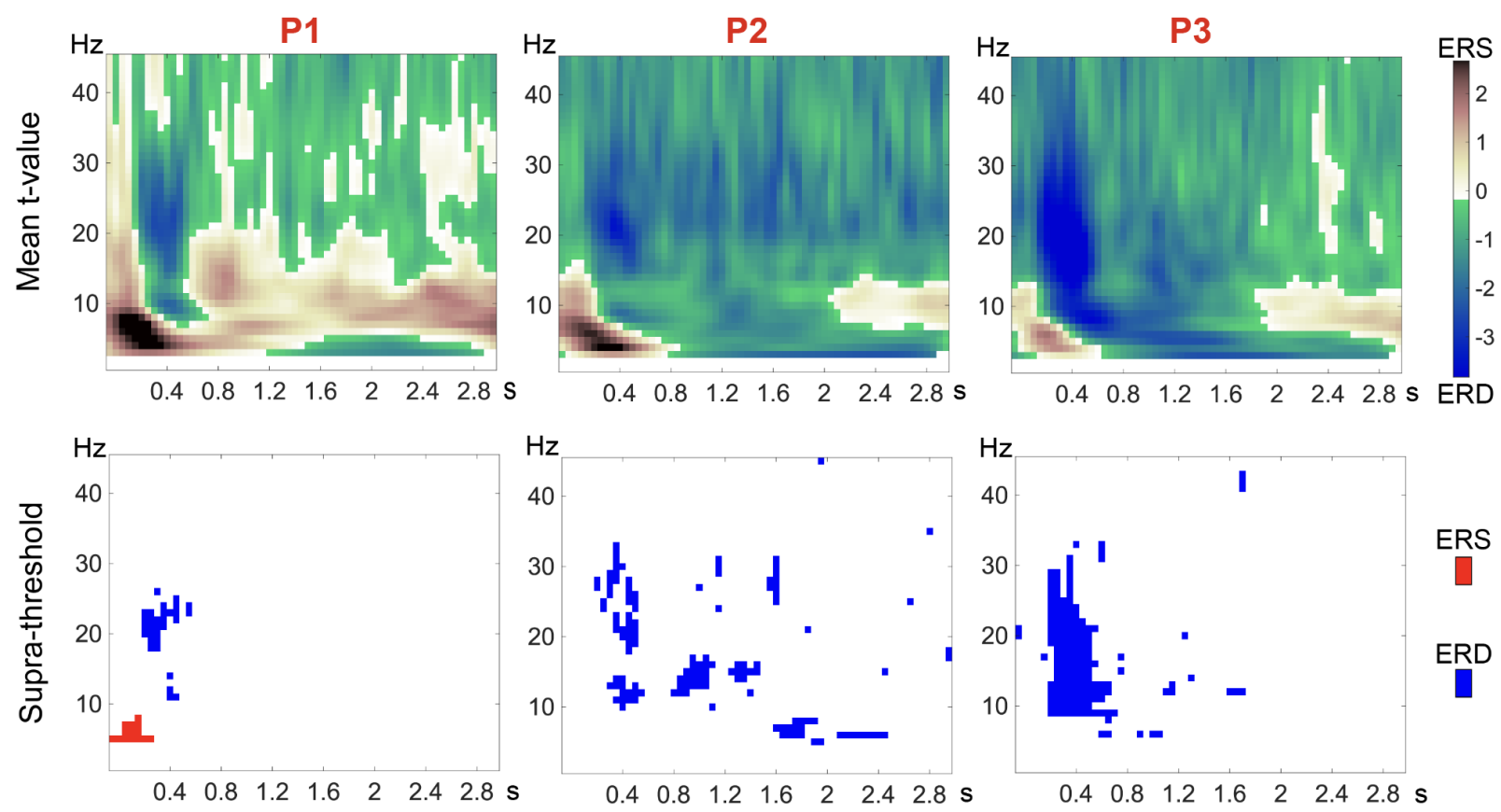

Figure 2: Time-frequency maps for the three parts of the SC paradigm. Wholebrain (average across all sources) power modulations associated with the first (P1, noun and determiner; Left), second (P2, part of predicate; Middle) and third part (P3, to be completed; Right) of the sentence. Top. Average of $t$-values of the statmax procedure without statistical masking, with a pink to dark red scale grading for positive (ERS) values and green to dark blue scale grading for negative (ERD) values. Bottom. 358 Time-frequency bins of significant ( $p<.05$ FWE corrected) ERS (red) or ERD (blue). 
359 3.2. Frequency band and time-resolved mapping associated with SC

360 For each sentence part, brain maps locating significant ERS and ERD in classic 361 frequency bands (theta: 4-7 Hz; alpha: 8-11 Hz; low-beta: $12-20 \mathrm{~Hz}$; high-beta: 21-30 Hz; 362 low-gamma: $31-45 \mathrm{~Hz}$ ) at any post-stimulus time are represented on Figure 3 along with 363 the corresponding LI measuring the left-right hemispheric asymmetry of significant ERS 364 or ERD. Their temporal development (in five consecutive 400 ms-long windows, followed 365 by a last longer window of $1000 \mathrm{~ms}$ ) are shown on Figure 4. Associated local maxima are 366 listed in Supplementary Tables 2 and 3. 


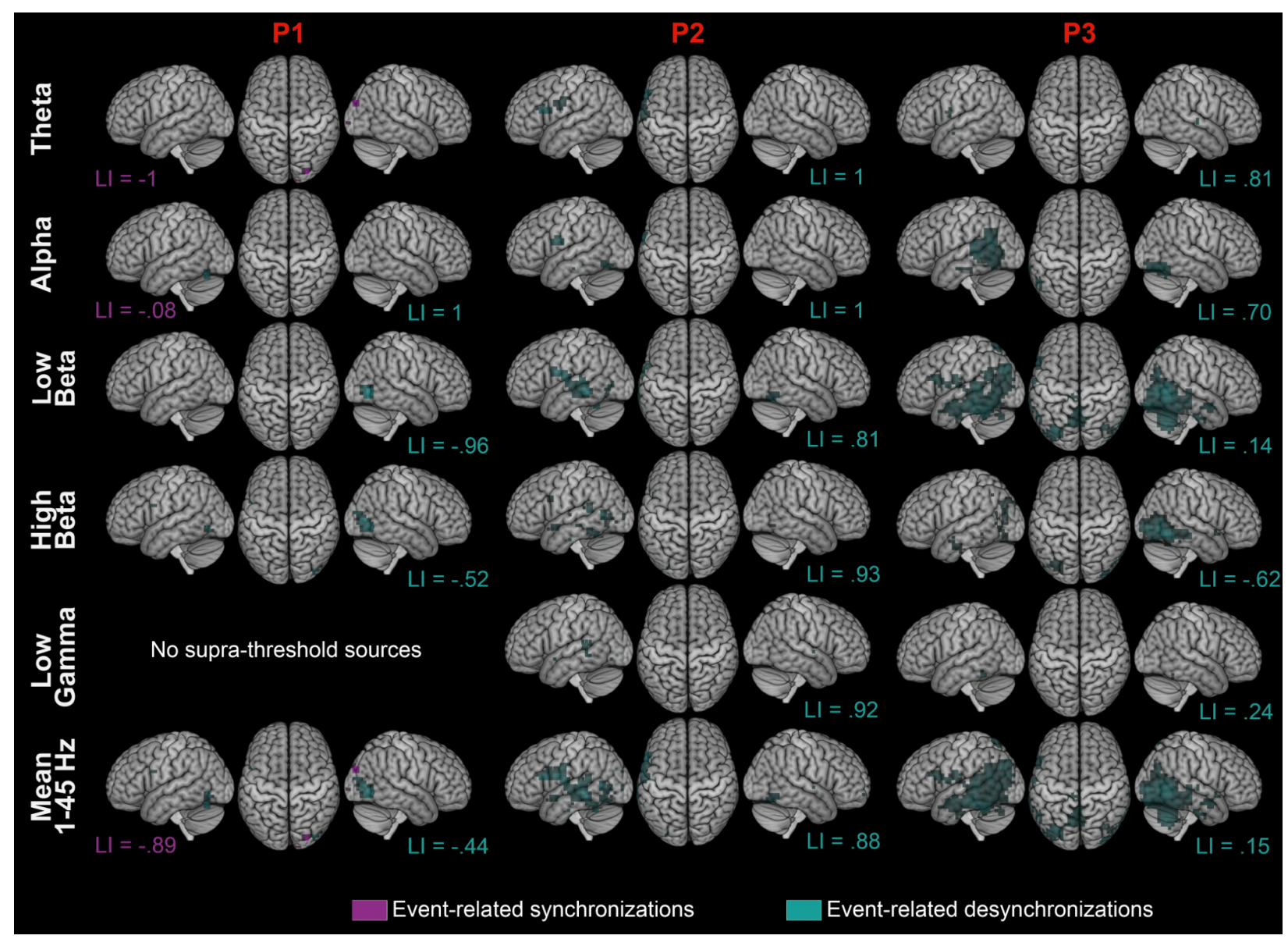

Figure 3: Brain maps showing frequency-dependent changes in neural activity

370 during the three parts of SC. Group-level maps of significant ( $p<.05 \mathrm{FWE}$ corrected)

371 ERS (magenta) and ERD (cyan) averaged across the whole post-stimulus period are

372 superimposed on surface projections (left lateral, top, right lateral) of the standard MNI

373 brain. The supratentorial lateralization indices (LI) derived by counting significant ERS

374 voxels (magenta font) and significant ERD voxels (cyan font) are also indicated (bottom

375 of left and right projections). The first (P1, noun and determiner; Left), second (P2, part

376 of predicate; Middle) and third (P3, to be completed; Right) parts are depicted in the

377 corresponding three columns. Rows correspond to frequency bands in which significant

378 ERS/ERD values were further averaged: theta $(4-7 \mathrm{~Hz})$, alpha $(8-11 \mathrm{~Hz})$, low beta (12-

$37920 \mathrm{~Hz})$, high beta (21-30 Hz), low gamma (30-45 Hz) and broadband average across

380 the whole frequency range $(1-45 \mathrm{~Hz})$. 


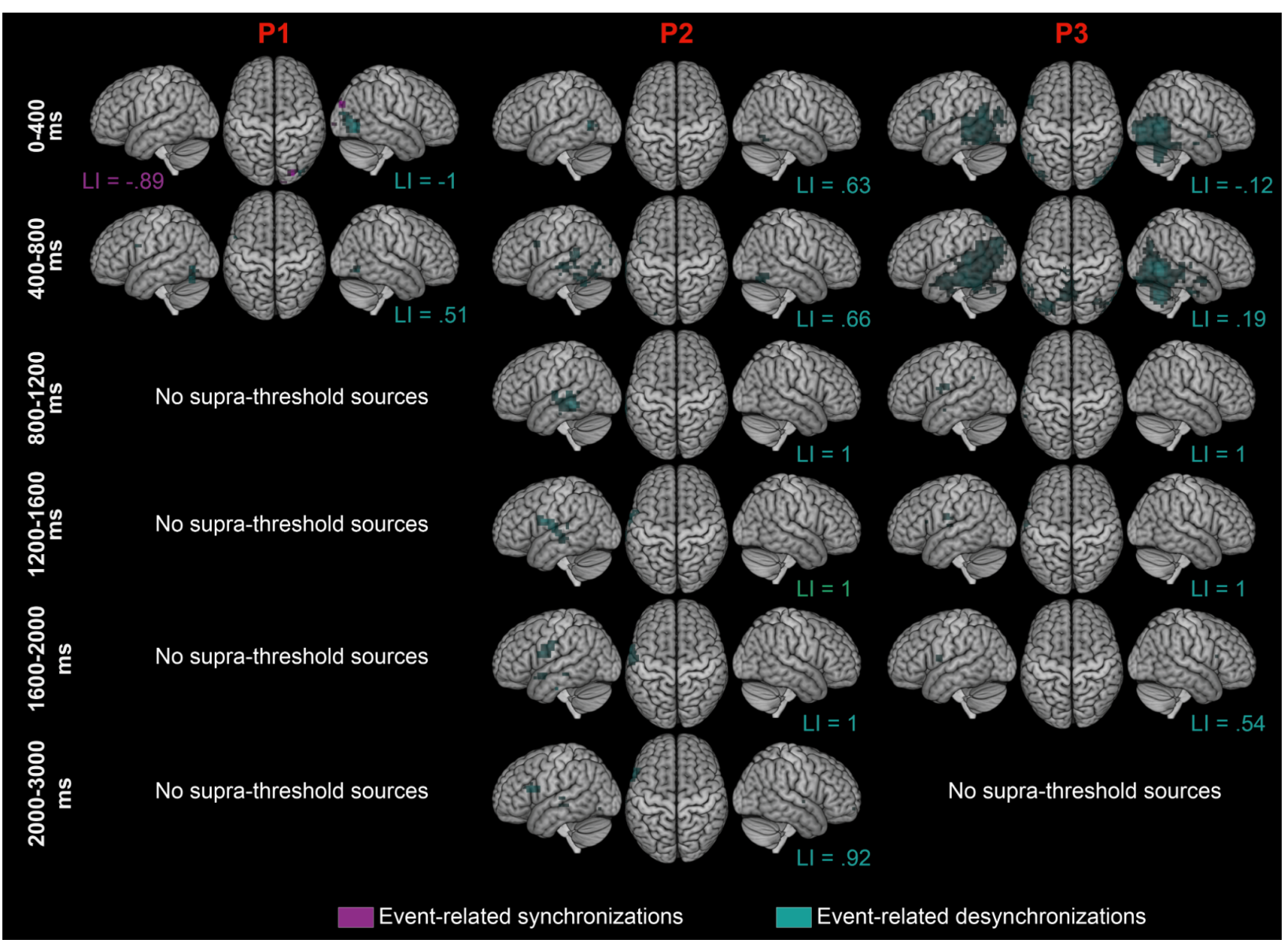

Figure 4: Brain maps showing time-dependent changes in neural activity during the three parts of SC. Group-level significant ( $p<.05$ FWE corrected) ERS (magenta) and ERD (cyan) averaged across the whole frequency range (1-45 Hz) superimposed on surface projections (left lateral, top, right lateral) of the standard MNI brain. The supratentorial lateralization indices (LI) derived by counting significant ERS voxels

388 (magenta font) and significant ERD voxels (cyan font) are indicated (bottom of left and 389 right projections). The first (P1: noun and determiner; Left), second (P2, part of 390 predicate; Middle) and third (P3, to be completed; Right) parts are depicted in the 391 corresponding three columns. The rows correspond to the temporal averages of 392 ERS/ERD within consecutive 400 ms-long windows except for the last row (over the last 393 second of the post-stimulus period). 
During the first part of the SC paradigm (P1; Figs. 3 and 4, left column), the first theta and alpha ERS occurring in the 0-400 ms time window were located in bilateral calcarine cortices (not visible on source projections but detailed in Supplementary Table 1-2) as well as in the right lateral occipital cortex, leading to a right-dominant LI. The lowbeta part of the ERD occurring in the same time window appeared in right lateral occipitotemporal and posterior fusiform regions, also leading to a right-dominant LI. Posteroinferior ERD became more bilateral and supported by high beta in the 400-800 ms timewindow, which also disclosed a small cluster of high-beta ERD in the left inferior frontal gyrus (IFG), leading to a left-dominant LI. No ERS/ERD was significant after 800 ms.

During the second part (P2; Figs. 3 and 4, middle column), the 0-800 ms time windows displayed bilateral beta ERD in the fusiform gyri with leftward dominance, along with left-sided beta ERD in the lateral occipito-temporal cortex and in the IFG. In the 8003000 ms time windows, broadband ERD were located in the lateral left temporal cortex (alpha, beta and low-gamma) and in left IFG and rolandic opercular regions (theta, alpha and beta; 1200-3000 ms time-windows), as well as the left posterior cingulate cortex (PCC; high-beta and low-gamma; 1200-1600 ms time-window; not visible on the projections). These ERD led to left-dominant $\mathrm{LI}$ for each time-window and frequency range.

The third part (P3; Figs. 3 and 4, right column) presented stronger and more extended ERD located in bilateral temporo-occipito-parietal areas, including more anterior aspects of the lateral temporal cortices. They occurred mostly in the 0-800 ms time windows and involved theta, alpha, beta and gamma frequency bands. In the 400$800 \mathrm{~ms}$ time window, beta-band ERD also emerged in the left posterior cingulate cortex (PCC) and precuneus, middle occipital gyri and angular gyri bilaterally and in the superior part of the cerebellum. Moreover, theta and beta ERD were observed in the left IFG and rolandic operculum from 0 to approximately 2000 ms post-stimulus. Finally, a late (1600$2000 \mathrm{~ms}$ ) and small gamma-band ERD was also present in the right IFG (not visible on the figure as it involved only a few brain sources). These ERDs led to left-dominant LI, except for the 0-800 ms time windows and beta and gamma-bands. 
423 Overall, in each sentence part, the alpha and beta frequency bands (Fig. 3, rows

$4242-4)$ accounted for the majority of the significant ERD in the wide-band, temporally

425 averaged maps (Fig. 3, last row).

426

427 3.3. Right vs. left comparative spectro-temporal neural dynamics in the ventral language

428 stream during SC

429 Among the three pairwise comparisons between the right and left components of 430 the ventral language stream, the only significant $(p=.0495$, with small-volume FWE and

431 Bonferroni correction) difference emerged in P2 in the alpha/low-beta bands (11-14 Hz)

432 in a cluster centered on 900-1050 ms. This difference originated from clearly more ERD

433 on the left, with a sustained 8-30 Hz ERD after the initial dominant ERD centered on

$434400 \mathrm{~ms}$, contrasting with a more subtle 8-20 Hz ERS trail on the right.

435 Infra-threshold analysis did not demonstrate prolonged ERD on the right side

436 compared to the left in P1 or P3. Indeed, in P1, the time-frequency dynamics was

437 similar to the global spectro-temporal dynamics of SC on both sides. In P3, the ERD

438 centered on 400 ms was actually slightly narrower. 

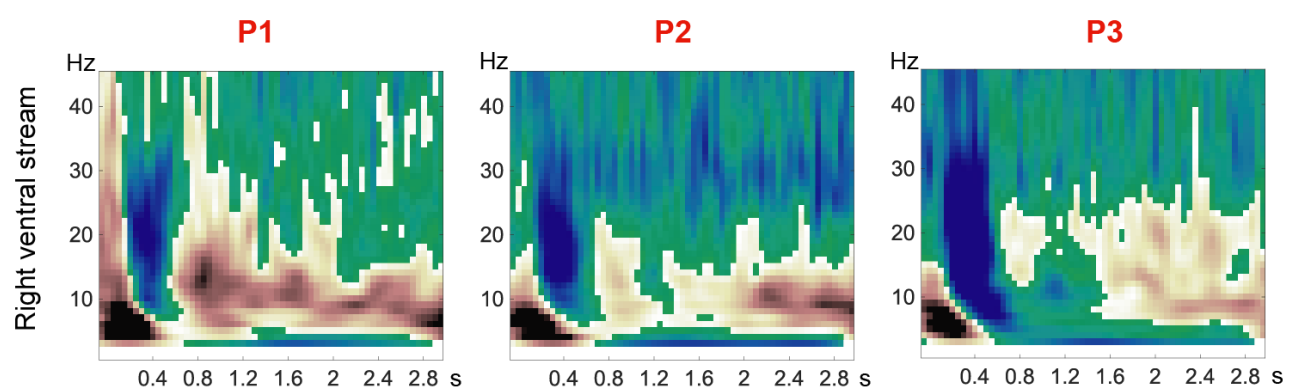

Regions-of-Interest
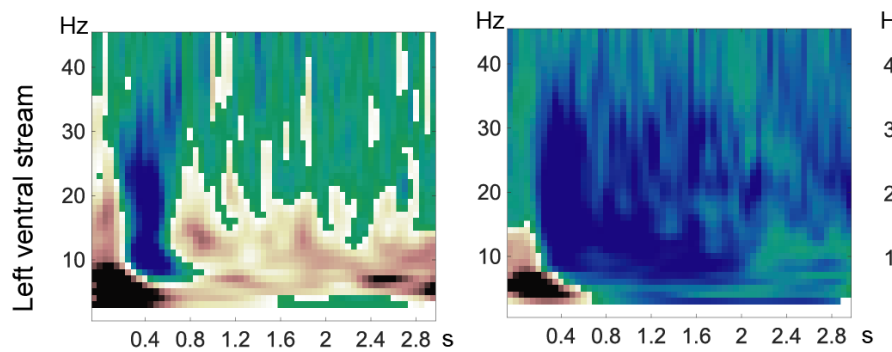

$\begin{array}{llllllll}0.4 & 0.8 & 1.2 & 1.6 & 2 & 2.4 & 2.8 \mathrm{~s}\end{array}$
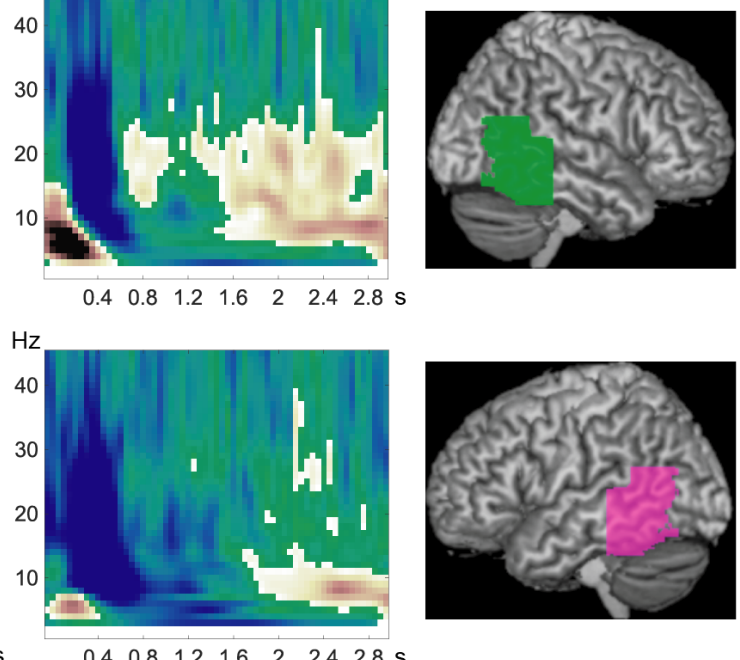

$\begin{array}{llllllll}0.4 & 0.8 & 1.2 & 1.6 & 2 & 2.4 & 2.8 \mathrm{~s}\end{array}$

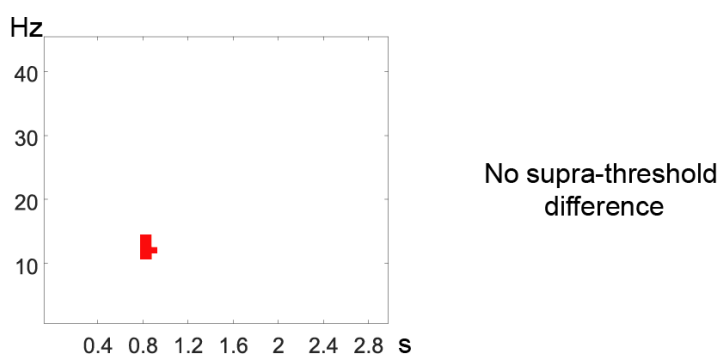

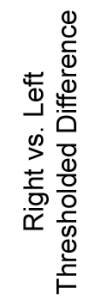

No supra-threshold difference

\begin{tabular}{|cccccccc|}
\hline Mean t-value & ERD & -3 & -2 & -1 & 0 & 1 & 2 \\
$\begin{array}{c}\text { Thresholded } \\
\text { difference }\end{array}$ & $\begin{array}{c}\text { Stronger ERD } \\
\text { on the Right }\end{array}$ & & & $\begin{array}{c}\text { Stronger ERS } \\
\text { on the Right }\end{array}$ \\
\hline
\end{tabular}

440 Figure 5: Right vs. left comparative spectro-temporal neural dynamics in the

441 ventral language stream during SC. The first (P1: noun and determiner), second (P2,

442 part of predicate) and third (P3, to be completed) parts are depicted in the

443 corresponding three columns. Time-frequency group-level $t$-statistics (without statistical

444 masking) averaged within a ROI (Region-of-Interest; rightmost column) are shown in

445 a pink to dark red scale grading the positive (ERS) and green to dark blue scale grading

446 the negative (ERD) $t$-values. Top. In the right component of the langage ventral stream

447 (green ROI). Middle. In its left component (pink ROI). Bottom. Pairwise right vs. left

448 comparison pairs ( $p<.05$ with small-volume FWE correction and additional Bonferroni

449 correction for the 3 comparisons considered), coded in red for a stronger ERD or

450 conversely, in blue for a stronger ERD on the right. 


\section{Discussion}

This MEG study demonstrates that SC elicits three successive significant neural response patterns characterized by distinct spectro-temporal signatures and anatomical locations for each sentence part: (i) an early (<300 ms post-stimulus) bilateral 4-10 Hz ERS in occipital cortices that is most conspicuous in P1; (ii) an 8-30 Hz ERD at about 400 ms post-stimulus involving the ventral language stream of both hemispheres as well as posterior nodes of the default mode network (DMN); and (iii) a late (> 800 ms poststimulus) 8-30 Hz ERD involving the left dorsal language stream observed in P2 and P3. Furthermore, the left ventral language stream displayed prolonged 8-30 Hz ERD from 800 ms post-stimulus during P2 compared to its right homolog which showed an 8-20 Hz ERS from $800 \mathrm{~ms}$ post-stimulus.

\subsection{First neural response pattern of SC: low-level visual processing}

The first neural response associated with SC was characterized by a 4-10 Hz ERS which was localized in the bilateral primary visual cortices and the right superior occipital gyrus during $\mathrm{P} 1$. A similar response was also observed in $\mathrm{P} 2$ and $\mathrm{P} 3$, but it did not reach statistical significance. This response is attributed to the visual processing of written stimuli given its rapid post-stimulus (0-300 ms) occurrence. This first response is in agreement with the timing and topography of previously reported MEG responses at play during reading and that are related to non-specific, pre-lexico-semantic visual features (Pammer et al., 2004; Tarkiainen et al., 1999; for a review, see Salmelin 2007).

4.2. Second neural response pattern of SC: bihemispheric semantic processing

The second response was associated with an ERD in the alpha-beta $(8-30 \mathrm{~Hz})$ frequency range centered at about $400 \mathrm{~ms}$ post-stimulus (starting at about 200-300 ms post-stimulus and lasting 300 to $400 \mathrm{~ms}$ ) in each part of the sentence, but most prominent during P2 and P3. It was located in the bilateral posterior inferior/middle temporal gyri, and also in the left IFG during P2.

The timing of this response probably relates to the previously described $\mathrm{N} 400 \mathrm{~m}$ evoked response associated with lexico-semantic processing (Halgren et al., 2002; 
482 Salmelin, 2007). As in the present study, this response has also been shown to involve 483 frontal brain areas, co-occurring as early as from 200-250 ms (Halgren et al., 2002; 484 Helenius, 1998; Pammer et al., 2004; Vartiainen et al., 2011). In accordance with previous 485 studies (Halgren et al., 2002; Hultén et al., 2019; Pammer et al., 2004), the observed 486 bilateral occipito-temporal ERD and the co-modulations occurring around $400 \mathrm{~ms}$ in the 487 left inferior frontal (in P1,2,3) and the temporal (left in P2 and bilateral in P3) areas are 488 jointly attributed to the same N400m response of SC, where phono-lexico-semantic 489 integration concomitantly occurs with the processing of visual words to conduct the reading process effectively.

491 The bi-hemispheric N400m ERD in the posterior temporal lobes consistently 492 colocalized with the ventral stream of language (Hickok \& Poeppel, 2007). It has been 493 suggested that word- and sentence-level comprehension rely on different processing 494 strategies in both hemispheres (Federmeier et al., 2008) and that specific right temporal 495 contribution is involved in sentence-level processing (Vigneau et al., 2010). However, at 496 this stage we did not find significant interhemispheric asymmetries in the spectral 497 signature that could suggest a differential contribution. In addition to the involvement of the ventral language stream, posterior parasagittal and lateral parietal cortices also presented ERD in the beta band at about 400 ms post500 stimulus, which is consistent with the concept of a distributed semantic network (Binder, 501 2015; Graves, 2010; Paunov et al., 2019; Seghier \& Price, 2012; Whitney et al., 2009), 502 involved in the semantic processes of sentences (as reviewed by Weiss \& Mueller, 2012).

503 Contrastingly, in fMRI, Graves (2010) and Whitney et al. (2009) relied on semantic tasks 504 being contrasted with other high-level language tasks (e.g., familiar highly meaningful 505 phrases vs. unfamiliar phrases with minimal meaning) to demonstrate a relative increase 506 in fMRI signal in the regions classically attributed to the default mode network (for a 507 review, see e.g., Raichle, 2015). These nodes are typically anticorrelated with the task at 508 hand and Seghier \& Price (2012) found that their degree of deactivation was dependent 509 on the semantic nature of the task. We here find a common beta-band ERD signature 510 throughout the whole extended semantic network, in distinction with the known 511 heterogeneity of its $\mathrm{fMRI}$ counterpart (activations and deactivations). The engagement of 
512 the posterior DMN may facilitate the retrieval and integration of relevant informational and

513 contextual content (Buckner et al., 2008) in order to select a relevant completion.

514 Of note, the beta-band ERD in the anterior lobe of the right cerebellum in P3 could

515 support the potential semantic role of the cerebellum in semantic language processing.

516 Right cerebellar contribution to the language function has been largely documented (for

517 reviews, see, e.g., Mariën et al., 2013; Price, 2012) and even used as a crossed cerebro-

518 cerebellar lateralization aid in a presurgical functional language mapping perspective

519 (Méndez Orellana et al., 2015). However, given the many language subprocesses it has

520 been associated with (Mariën et al., 2013), the ERD in the right cerebellum might reflect

521 its role as a general contributor/modulator of higher cognitive processes rather than

522 representing another specific node of the distributed semantic network.

4.3. Third neural response pattern of SC: left-sided dorsal stream integration

The third and last neural response pattern was characterized by an alpha-beta ERD in P2 and P3, taking place from $>800 \mathrm{~ms}$ post-stimulus. Contrary to previous responses, this ERD, most clearly demonstrated in P2, presented a clear leftward dominance (LIERD = .54 to 1 ) and mainly involved the left fronto-temporal regions. These areas (IFG, superior temporal gyrus) are topographically consistent with the key regions of the classical Broca-Wernicke-Lichtheim-Geschwind model (Tremblay \& Dick, 2016) and of the more recently proposed MUC (Memory, Unification, Control) model (Hagoort, 2013). Information processed in the previous N400 step might be integrated during these long-lasting ERD within the left hemisphere, dominant for language processing (TzourioMazoyer et al., 2017), to be further semantically and syntactically unified, and to allow control (Hagoort, 2013) and selection of the required words to properly complete the sentence. They are also anatomically in line with the left-dominant dorsal stream of the dual stream model (Hickok \& Poeppel, 2007), including the revisited, speech production Wernicke's area (Binder, 2015). They might therefore also reflect some level of production and phonological processes (Vigneau et al., 2006), such as verbal working memory to maintain the sentence content (Meltzer et al., 2017).

In the ventral stream, no whole-brain suprathreshold modulation was demonstrated at this stage. However, focused ROI analyses showed a sustained alpha- 
543 beta ERD on the left, contrasting with moderate ERS in those frequency bands on the 544 right in P2 and P3 (the right vs. left difference reaching significance only in P2). 545 Considering that alpha-beta bands ERS may be considered as an electrophysiological 546 correlate of a deactivated/inhibited cortical area (for reviews, see, e.g., Klimesch et al., 547 2007; Pfurtscheller, 2001), we here suggest a left-to-right interhemispheric inhibition 548 (Tzourio-Mazoyer et al., 2017) where the left hemisphere would recover its dominance 549 for efficient left intra-hemispheric integration (Gotts et al., 2013), through a left-to-right 550 "top-down" inhibition by means of alpha-beta modulations (Fries, 2015). Indeed, the 551 genetic, developmental, structural and functional factors (for reviews, see e.g., Stephan 552 et al., 2007; Tzourio-Mazoyer et al., 2017) leading to left hemisphere specialization for 553 language, might position this hemisphere as the actual higher order language hemisphere 554 for subsequent SC process, endowed with the control component of the MUC model 555 (Hagoort, 2013). We therefore did not find signs of sustained engagement of the right 556 hemisphere that could indicate retention of multiple meanings for prolonged periods of 557 time (Kircher et al., 2001) or integration of information over longer timescales as in 558 auditory speech processing (Hickok \& Poeppel, 2007).

4.4. Neural processing in the ventral and dorsal streams of the language network during $\underline{\mathrm{SC}}$ is dominated by transient alpha-beta frequency band power decrease

Spectral specialization in language processing has been suggested for different frequency bands due to selectivity to certain linguistic manipulations (for a review, see, e.g., Meyer, 2017). It can also be suspected given the observation of a spatio-spectral pattern in the language network during task (Goto et al., 2011) or at rest (Coolen et al., 2020), whereby lower frequency bands (theta, alpha) tend to localize ventrally and posteriorly while higher frequency bands (beta, gamma) are more dorsal and anterior, in their modulations or connectivity.

However, in this study, alpha and beta ERD represented the main neural activity modulations observed within the whole language network. The main alpha-beta-band 571 spectrum in language-related neural processes found here is in agreement with the 572 prominence of the ERD in those two frequency bands reported in previous MEG language 573 studies (Goto et al., 2011; Lam et al., 2016; Piai et al., 2015), and with the concept of a 
574 broadband alpha-beta (8-30 Hz) ERD in sentence processing (Kielar et al., 2015; Meltzer 575 et al., 2017; Meltzer \& Braun, 2011). The alpha (e.g., Obleser \& Weisz, 2012; Wang et 576 al., 2018) and beta (e.g., Piai et al., 2015; Weiss \& Mueller, 2012) frequency bands have 577 been shown to be involved in language processes in various experimental designs and 578 to serve as the main channels for interactions within the language network (Schoffelen et 579 al., 2017). Beyond information transfer (Buzsáki \& Draguhn, 2004), the observed 580 coordinated alpha-beta ERD constituting the response patterns in the extended semantic 581 network and in the dorsal stream of language might also reflect the formation of specific 582 dynamic workspaces (Lopes da Silva, 2013), for efficient and sustained language 583 processing. In addition, a spatial correlation was previously shown between task-related 584 ERD in alpha-beta bands and the fMRI task-related changes (Hall et al., 2014; Mukamel, 585 2005), particularly in language tasks (Singh et al., 2002). 


\subsection{Limitations}

In contrast with some previous sentence-level paradigms (e.g., Kielar et al., 2015; Meltzer et al., 2017), this study did not use specific linguistic manipulations. In particular, the lack of controlled semantic modulations (e.g., theory-of-mind, semantic complexity) did not allow for the refinement of the relative contribution of the constituent parts of the observed extended semantic network, especially that of the posterior nodes of the DMN. We also did not use standardized sentences (e.g., Kircher et al., 2001; Wilson et al., 2017) nor recorded the completions given by the subjects. Moreover, our design ( 3 second-long variable length stimuli) did not allow for a fine-grained temporal analysis (e.g., Halgren et al., 2002; Hultén et al., 2019). We instead provided a continuous, detailed neuromagnetic overview of the neural dynamics occurring during a classic nonstandardized SC paradigm.

Finally, the rather limited population, the prior-free whole-brain analysis and the stringent maximum statistics that we used might have impeded statistical power. For instance, the immediate post-stimulus lower-level visual processing step (described in 4.1) reached statistical significance only in $P 1$. In the other two parts of the sentence (P2, $\mathrm{P} 3)$, this approach might be solely picking up the maximum difference from baseline driven by the language-related ERD. This probably includes the temporal modulations around $400 \mathrm{~ms}$ associated with the increasing semantic load and N400m effect (Halgren et al., 2002), that remained infra-threshold in P1. Similarly, the relative lack of significant deviations from baseline observed in the theta and gamma bands could be partly due to the biased sensitivity of our statistical approach to the stronger and most consistent ERD in alpha and beta bands. Conversely, the spectro-temporal modulations we reported are highly likely to represent true neural responses. The use of stringent maximum statistics also ensures the robustness of the observed neural activity modulations. 


\section{Conclusions}

This study elucidates the neural dynamics that accompany the SC process, consisting of three successive neural response patterns associated with distinct spatial and spectro-temporal characteristics: an early low-level visual response; an intermediate bihemispheric semantic processing within an extended semantic network; a late lefthemisphere dorsal language stream integration.

We brought critical insight into the differential contribution of the ventral language stream, where the right-sided contribution is more transient and associated with some signs of subsequent inhibition. Furthermore, we demonstrated a common fingerprint throughout an extended semantic network. This shared signature is in contradistinction with its known heterogeneous hemodynamics (i.e., task-correlation of fMRI signal in temporal areas versus anticorrelation in the DMN). Therefore, this study may encourage more MEG research in sentence-level paradigms and their engagement of the extended semantic network. MEG-fMRI correspondence studies are also needed to elucidate the apparent heterogeneity of the neurovascular coupling within that network.

This adaptation of the fMRI SC paradigm to MEG paves the way for neuromagnetic preoperative language mapping in neurosurgical patients, free of neurovascular coupling issues (see e.g., Pak et al., 2017). Thanks to the dynamic dimension brought by MEG in SC, this may help in the identification of regions that need to be preserved among all activated regions disclosed by the static mapping fMRI provides. It also opens new perspectives in understanding the physiopathology of language alterations in various brain disorders. For instance, sentence completion tests have been administered to evaluate cognition in neurodegenerative diseases, such as Alzheimer's disease (Kim \& Thompson, 2004; Martyr et al., 2019) Parkinson's disease (Martyr et al., 2019; Siquier \& Andrés, 2021) and amyotrophic lateral sclerosis (Abrahams et al., 2005). MEG should

637 bring novel insights in the spectro-temporal correlates of previously reported abnormalities. 


\section{CRediT author statement}

T. Coolen: Conceptualization, Software, Formal Analysis, Investigation, Writing Original Draft, Visualization. A.M. Dumitrescu: Conceptualization, Formal Analysis,

643 Investigation, Writing - Review and Editing, Visualization. V. Wens: Methodology,

644 Software, Formal Analysis, Resources, Writing - Review and Editing. M. Bourguignon:

645 Software, Review and Editing. G.L. Gómez: Methodology, Software, Resources. A.

646 Rovai: Software, Resources. N. Sadeghi: Writing - Review and Editing, Supervision. C.

647 Urbain: Writing - Review and Editing, Supervision. S. Goldman: Writing - Review and

648 Editing, Supervision, Project Administration, Funding Acquisition. X. De Tiège:

649 Conceptualization, Writing - Review and Editing, Project Administration, Funding

650 Acquisition.

\section{Acknowledgements}

Tim Coolen (MD, PhD student) is Clinical Master Specialist Applicant to a PhD at the Fonds de la Recherche Scientifique (FRS-FNRS, Brussels, Belgium). Alexandru Dumitrescu is supported by the Fonds Erasme (Research Convention "Les Voies du Savoir", Brussels, Belgium). Gustavo Lucena Gómez was supported by the Excellence of Science "MEMODYN" of the Fonds de la Recherche Scientifique (FRS-FNRS, Brussels, Belgium). Xavier De Tiège is a Postdoctorate Clinical Master Specialist at the FRS-FNRS (Brussels, Belgium). This study and the MEG project at the CUB Hôpital Erasme are financially supported by the Fonds Erasme (Research Convention "Les Voies du Savoir", Brussels, Belgium). Funding sources had no involvement in the present study. Declarations of interest: none.

We extend our gratitude to Thomas Amighi (Master Student at the Faculty of Psychology, Université libre de Bruxelles) for collecting the behavioral data presented in the Supplementary Materials. 


\section{References}

Abrahams, S., Leigh, P. N., \& Goldstein, L. H. (2005). Cognitive change in ALS: A prospective study. Neurology, 64(7), 1222-1226. https://doi.org/10.1212/01.WNL.0000156519.41681.27

Amaro, E., \& Barker, G. J. (2006). Study design in fMRI: Basic principles. Brain and Cognition, 60(3), 220-232. https://doi.org/10.1016/j.bandc.2005.11.009

Ashtari, M., Perrine, K., Elbaz, R., Syed, U., Thaden, E., Mcllree, C., Dolgoff-Kaspar, R., Clarke, T., Diamond, A., \& Ettinger, A. (2005). Mapping the functional anatomy of sentence comprehension and application to presurgical evaluation of patients with brain tumor. AJNR. American Journal of Neuroradiology, 26(6), 1461-1468.

Barnett, A., Marty-Dugas, J., \& McAndrews, M. P. (2014). Advantages of sentence-level fMRI language tasks in presurgical language mapping for temporal lobe epilepsy. Epilepsy \& Behavior, 32, 114 120. https://doi.org/10.1016/j.yebeh.2014.01.010

Binder, J. R. (2015). The Wernicke area: Modern evidence and a reinterpretation. Neurology, 85(24), 2170-2175. https://doi.org/10.1212/WNL.0000000000002219

Black, D. F., Vachha, B., Mian, A., Faro, S. H., Maheshwari, M., Sair, H. I., Petrella, J. R., Pillai, J. J., \& Welker, K. (2017). American society of functional neuroradiology-recommended fMRI paradigm algorithms for presurgical language assessment. American Journal of Neuroradiology, 38(10), E65-E73. https://doi.org/10.3174/ajnr.A5345

Buckner, R. L., Andrews-Hanna, J. R., \& Schacter, D. L. (2008). The Brain's Default Network: Anatomy, Function, and Relevance to Disease. Annals of the New York Academy of Sciences, 1124(1), 138. https://doi.org/10.1196/annals.1440.011

Buzsáki, G., \& Draguhn, A. (2004). Neuronal oscillations in cortical networks. Science, 304(5679), 19261929. https://doi.org/10.1126/science.1099745

Coolen, T., Wens, V., Vander Ghinst, M., Mary, A., Bourguignon, M., Naeije, G., Peigneux, P., Sadeghi, N., Goldman, S., \& De Tiège, X. (2020). Frequency-Dependent Intrinsic Electrophysiological Functional Architecture of the Human Verbal Language Network. Frontiers in Integrative Neuroscience, 14, 27. https://doi.org/10.3389/fnint.2020.00027

Dale, A. M., \& Sereno, M. I. (1993). Improved Localizadon of Cortical Activity by Combining EEG and MEG with MRI Cortical Surface Reconstruction: A Linear Approach. Journal of Cognitive Neuroscience, 5(2), 162-176. https://doi.org/10.1162/jocn.1993.5.2.162

De Tiège, X., de Beeck, M. O., Funke, M., Legros, B., Parkkonen, L., Goldman, S., \& Van Bogaert, P. (2008). Recording epileptic activity with MEG in a light-weight magnetic shield. Epilepsy Research, 82(2-3), 227-231. https://doi.org/10.1016/J.EPLEPSYRES.2008.08.011

Federmeier, K. D., Wlotko, E. W., \& Meyer, A. M. (2008). What's 'Right' in Language Comprehension: Event-Related Potentials Reveal Right Hemisphere Language Capabilities. Language and Linguistics Compass, 2(1), 1-17. https://doi.org/10.1111/j.1749-818X.2007.00042.x

Fischl, B. (2012). FreeSurfer. Neuroimage, 62(2), 774-781. https://doi.org/10.1016/j.neuroimage.2012.01.021.FreeSurfer

Fries, P. (2015). Rhythms for Cognition: Communication through Coherence. Neuron, 88(1), 220-235. https://doi.org/10.1016/j.neuron.2015.09.034

Goto, T., Hirata, M., Umekawa, Y., Yanagisawa, T., Shayne, M., Saitoh, Y., Kishima, H., Yorifuji, S., \& Yoshimine, T. (2011). Frequency-dependent spatiotemporal distribution of cerebral oscillatory changes during silent reading: A magnetoencephalograhic group analysis. Neurolmage, 54(1), 560-567. https://doi.org/10.1016/j.neuroimage.2010.08.023

Gotts, S. J., Jo, H. J., Wallace, G. L., Saad, Z. S., Cox, R. W., \& Martin, A. (2013). Two distinct forms of functional lateralization in the human brain. Proceedings of the National Academy of Sciences, 110(36), E3435-E3444. https://doi.org/10.1073/pnas.1302581110 
716

717

718

719

720

721

722

723

724

725

726

727

728

729

730

731

732

733

734

735

736

737

738

739

740

741

742

743

744

745

746

747

748

749

750

751

752

753

754

755

756

757

758

759

760

761

762

763

764

Gramfort, A., Luessi, M., Larson, E., Engemann, D., Strohmeier, D., Brodbeck, C., Parkkonen, L., \& Hämäläinen, M. (2014). MNE software for processing MEG and EEG data. Neuroimage, 86, 446460. https://doi.org/10.1016/j.neuroimage.2013.10.027

Graves, W. W. (2010). Neural correlates of implicit and explicit combinatorial semantic processing. 9.

Gross, J., Baillet, S., Barnes, G. R., Henson, R. N., Hillebrand, A., Jensen, O., Jerbi, K., Litvak, V., Maess, B., Oostenveld, R., Parkkonen, L., Taylor, J. R., van Wassenhove, V., Wibral, M., \& Schoffelen, J. M. (2013). Good practice for conducting and reporting MEG research. Neurolmage, 65, 349-363. https://doi.org/10.1016/j.neuroimage.2012.10.001

Hagoort, P. (2013). MUC (memory, unification, control) and beyond. Frontiers in Psychology, 4(JUL), 113. https://doi.org/10.3389/fpsyg.2013.00416

Halgren, E., Dhond, R. P., Christensen, N., Van Petten, C., Marinkovic, K., Lewine, J. D., \& Dale, A. M. (2002). N400-like magnetoencephalography responses modulated by semantic context, word frequency, and lexical class in sentences. Neurolmage, 17(3), 1101-1116. https://doi.org/10.1006/nimg.2002.1268

Hall, E. L., Robson, S. E., Morris, P. G., \& Brookes, M. J. (2014). The relationship between MEG and fMRI. Neurolmage. https://doi.org/10.1016/j.neuroimage.2013.11.005

Helenius, P. (1998). Distinct time courses of word and context comprehension in the left temporal cortex. Brain, 121(6), 1133-1142. https://doi.org/10.1093/brain/121.6.1133

Hickok, G., \& Poeppel, D. (2007). The cortical organization of speech processing. Nature Reviews. Neuroscience, 8(5), 393-402. https://doi.org/10.1038/nrn2113

Hultén, A., Schoffelen, J.-M., Uddén, J., Lam, N. H. L., \& Hagoort, P. (2019). How the brain makes sense beyond the processing of single words - An MEG study. Neurolmage, 186, 586-594. https://doi.org/10.1016/j.neuroimage.2018.11.035

Hyvärinen, A., \& Oja, E. (2000). Independent Component Analysis: Algorithms and Applications. In Neural Networks (Vol. 13, Issue 5, pp. 411-430).

Kielar, A., Panamsky, L., Links, K. A., \& Meltzer, J. A. (2015). Localization of electrophysiological responses to semantic and syntactic anomalies in language comprehension with MEG. Neurolmage, 105, 507-524. https://doi.org/10.1016/j.neuroimage.2014.11.016

Kim, M., \& Thompson, C. K. (2004). Verb deficits in Alzheimer's disease and agrammatism: Implications for lexical organization. Brain and Language, 88(1), 1-20. https://doi.org/10.1016/S0093934X(03)00147-0

Kircher, T. T. J., Brammer, M., Tous Andreu, N., Williams, S. C. R., \& McGuire, P. K. (2001). Engagement of right temporal cortex during processing of linguistic context. Neuropsychologia, 39(8), 798809. https://doi.org/10.1016/S0028-3932(01)00014-8

Klimesch, W., Sauseng, P., \& HansImayr, S. (2007). EEG alpha oscillations: The inhibition-timing hypothesis. Brain Research Reviews, 53(1), 63-88. https://doi.org/10.1016/j.brainresrev.2006.06.003

Lam, N. H. L., Schoffelen, J.-M., Uddén, J., Hultén, A., \& Hagoort, P. (2016). Neural activity during sentence processing as reflected in theta, alpha, beta, and gamma oscillations. Neurolmage, 142, 43-54. https://doi.org/10.1016/j.neuroimage.2016.03.007

Lopes da Silva, F. (2013). EEG and MEG: Relevance to neuroscience. Neuron, 80(5), 1112-1128. https://doi.org/10.1016/j.neuron.2013.10.017

Mariën, P., Ackermann, H., Adamaszek, M., Barwood, C. H. S., Beaton, A., Desmond, J., De Witte, E., Fawcett, A. J., Hertrich, I., Küper, M., Leggio, M., Marvel, C., Molinari, M., Murdoch, B. E., Nicolson, R. I., Schmahmann, J. D., Stoodley, C. J., Thürling, M., Timmann, D., ... Ziegler, W. (2013). Consensus Paper: Language and the Cerebellum: an Ongoing Enigma. The Cerebellum. https://doi.org/10.1007/s12311-013-0540-5

Martyr, A., Boycheva, E., \& Kudlicka, A. (2019). Assessing inhibitory control in early-stage Alzheimer's and Parkinson's disease using the Hayling Sentence Completion Test. Journal of 
Neuropsychology, 13(1), 67-81. https://doi.org/10.1111/jnp.12129

Meltzer, J. A., \& Braun, A. R. (2011). An EEG-MEG dissociation between online syntactic comprehension and post hoc reanalysis. Frontiers in Human Neuroscience, 5(FEBRUARY), 1-15. https://doi.org/10.3389/fnhum.2011.00010

Meltzer, J. A., Kielar, A., Panamsky, L., Links, K. A., Deschamps, T., \& Leigh, R. C. (2017). Electrophysiological signatures of phonological and semantic maintenance in sentence repetition. Neurolmage, 156, 302-314. https://doi.org/10.1016/j.neuroimage.2017.05.030

Méndez Orellana, C., Visch-Brink, E., Vernooij, M., Kalloe, S., Satoer, D., Vincent, A., Van Der Lugt, A., \& Smits, M. (2015). Crossed Cerebrocerebellar Language Lateralization: An Additional Diagnostic Feature for Assessing Atypical Language Representation in Presurgical Functional MR Imaging. American Journal of Neuroradiology, 36(3), 518-524. https://doi.org/10.3174/ajnr.A4147

Meyer, L. (2017). The neural oscillations of speech processing and language comprehension: State of the art and emerging mechanisms. European Journal of Neuroscience, 1-13. https://doi.org/10.1111/ejn.13748

Mukamel, R. (2005). Coupling Between Neuronal Firing, Field Potentials, and fMRI in Human Auditory Cortex. Science, 309(5736), 951-954. https://doi.org/10.1126/science.1110913

Obleser, J., \& Weisz, N. (2012). Suppressed alpha oscillations predict intelligibility of speech and its acoustic details. Cerebral Cortex, 22(11), 2466-2477. https://doi.org/10.1093/cercor/bhr325

Oldfield, R. C. (1971). The assessment and analysis of handedness: The Edinburgh inventory. Neuropsychologia, 9(1), 97-113. https://doi.org/10.1016/0028-3932(71)90067-4

Oostenveld, R., Fries, P., Maris, E., \& Schoffelen, J. M. (2011). FieldTrip: Open source software for advanced analysis of MEG, EEG, and invasive electrophysiological data. Computational Intelligence and Neuroscience, 2011. https://doi.org/10.1155/2011/156869

Pak, R. W., Hadjiabadi, D. H., Senarathna, J., Agarwal, S., Thakor, N. V., Pillai, J. J., \& Pathak, A. P. (2017). Implications of neurovascular uncoupling in functional magnetic resonance imaging (fMRI) of brain tumors. Journal of Cerebral Blood Flow \& Metabolism, 37(11), 3475-3487. https://doi.org/10.1177/0271678X17707398

Pammer, K., Hansen, P. C., Kringelbach, M. L., Holliday, I., Barnes, G., Hillebrand, A., Singh, K. D., \& Cornelissen, P. L. (2004). Visual word recognition: The first half second. Neurolmage, 22(4), 1819-1825. https://doi.org/10.1016/j.neuroimage.2004.05.004

Pascual-Marqui, R. D. (2002). Standardized low resolution brain electromagnetic. Clinical Pharmacology, 16.

Paunov, A. M., Blank, I. A., \& Fedorenko, E. (2019). Functionally distinct language and Theory of Mind networks are synchronized at rest and during language comprehension. Journal of Neurophysiology, 121(4), 1244-1265. https://doi.org/10.1152/jn.00619.2018

Petrella, J. R., Shah, L. M., Harris, K. M., Friedman, A. H., George, T. M., Sampson, J. H., Pekala, J. S., \& Voyvodic, J. T. (2006). Preoperative functional MR imaging localization of language and motor areas: Effect on therapeutic decision making in patients with potentially resectable brain tumors. Radiology, 240(3), 793-802. https://doi.org/10.1148/radiol.2403051153

Pfurtscheller, G. (2001). Functional brain imaging based on ERD/ERS. Vision Research, 41(10-11), 1257-1260. https://doi.org/10.1016/S0042-6989(00)00235-2

Piai, V., Roelofs, A., Rommers, J., \& Maris, E. (2015). Beta oscillations reflect memory and motor aspects of spoken word production. Human Brain Mapping, 36(7), 2767-2780. https://doi.org/10.1002/hbm.22806

Price, C. J. (2012). A review and synthesis of the first 20years of PET and fMRI studies of heard speech, spoken language and reading. Neurolmage, 62(2), 816-847. https://doi.org/10.1016/j.neuroimage.2012.04.062

Raichle, M. E. (2015). The Brain's Default Mode Network. Annual Review of Neuroscience, 38(1), 433447. https://doi.org/10.1146/annurev-neuro-071013-014030 
814

815

816

817

818

819

820

821

822

823

824

825

826

827

828

829

830

831

832

833

834

835

836

837

838

839

840

841

842

843

844

845

846

847

848

849

850

851

852

853

854

855

856

857

858

859

860

861

862

Rorden, C., \& Brett, M. (2000). Stereotaxic Display of Brain Lesions. Behavioural Neurology, 12(4), 191200. https://doi.org/10.1155/2000/421719

Salek, K. E., Hassan, I. S., Kotrotsou, A., Abrol, S., Faro, S. H., Mohamed, F. B., Zinn, P. O., Wei, W., Li, N., Kumar, A. J., Weinberg, J. S., Wefel, J. S., Kesler, S. R., Liu, H. L. A., Hou, P., Stafford, R. J., Prabhu, S., Sawaya, R., \& Colen, R. R. (2017). Silent Sentence Completion Shows Superiority Localizing Wernicke's Area and Activation Patterns of Distinct Language Paradigms Correlate with Genomics: Prospective Study. Scientific Reports, 7(1), 1-8. https://doi.org/10.1038/s41598017-11192-2

Salmelin, R. (2007). Clinical neurophysiology of language: The MEG approach. Clinical Neurophysiology, 118(2), 237-254. https://doi.org/10.1016/j.clinph.2006.07.316

Saur, D., Kreher, B. W., Schnell, S., Kümmerer, D., Kellmeyer, P., Vry, M.-S., Umarova, R., Musso, M., Glauche, V., Abel, S., Huber, W., Rijntjes, M., Hennig, J., \& Weiller, C. (2008). Ventral and dorsal pathways for language. Proceedings of the National Academy of Sciences of the United States of America, 105(46), 18035-18040. https://doi.org/10.1073/pnas.0805234105

Schoffelen, J.-M., Hultén, A., Lam, N., Marquand, A. F., Uddén, J., \& Hagoort, P. (2017). Frequencyspecific directed interactions in the human brain network for language. Proceedings of the National Academy of Sciences, 114(30), 8083-8088. https://doi.org/10.1073/pnas.1703155114

Seghier, M. L., \& Price, C. J. (2012). Functional heterogeneity within the default network during semantic processing and speech production. Frontiers in Psychology, 3(AUG), 1-16. https://doi.org/10.3389/fpsyg.2012.00281

Singh, K. D., Barnes, G. R., Hillebrand, A., Forde, E. M. E., \& Williams, A. L. (2002). Task-related changes in cortical synchronization are spatially coincident with the hemodynamic response. Neurolmage, 16(1), 103-114. https://doi.org/10.1006/nimg.2001.1050

Siquier, A., \& Andrés, P. (2021). Cognitive and Behavioral Inhibition Deficits in Parkinson's Disease: The Hayling Test as a Reliable Marker. Frontiers in Aging Neuroscience, 12, 621603. https://doi.org/10.3389/fnagi.2020.621603

Stephan, K. E., Fink, G. R., \& Marshall, J. C. (2007). Mechanisms of hemispheric specialization: Insights from analyses of connectivity. Neuropsychologia, 45(2), 209-228.

https://doi.org/10.1016/j.neuropsychologia.2006.07.002

Stippich, C., Rapps, N., Dreyhaupt, J., Durst, A., Kress, B., Nennig, E., Tronnier, V. M., \& Sartor, K. (2007). Localizing and lateralizing language in patients with brain tumors: Feasibility of routine preoperative functional MR imaging in 81 consecutive patients. Radiology, 243(3), 828-836. https://doi.org/10.1148/radiol.2433060068

Tarkiainen, A., Helenius, P., Hansen, P. C., Cornelissen, P. L., \& Salmelin, R. (1999). Dynamics of letter string perception in the human occipitotemporal cortex. Brain, 122(11), 2119-2132. https://doi.org/10.1093/brain/122.11.2119

Taulu, S., Simola, J., \& Kajola, M. (2005). Applications of the signal space separation method. IEEE Transactions on Signal Processing, 53(9), 3359-3372.

Tremblay, P., \& Dick, A. S. (2016). Broca and Wernicke are dead, or moving past the classic model of language neurobiology. Brain and Language, 162(December), 60-71. https://doi.org/10.1016/j.bandl.2016.08.004

Tzourio-Mazoyer, N., Landeau, B., Papathanassiou, D., Crivello, F., Etard, O., Delcroix, N., Mazoyer, B., \& Joliot, M. (2002). Automated anatomical labeling of activations in SPM using a macroscopic anatomical parcellation of the MNI MRI single-subject brain. Neurolmage, 15(1), 273-289. https://doi.org/10.1006/nimg.2001.0978

Tzourio-Mazoyer, N., Perrone-Bertolotti, M., Jobard, G., Mazoyer, B., \& Baciu, M. (2017). Multi-factorial modulation of hemispheric specialization and plasticity for language in healthy and pathological conditions: A review. Cortex, 86, 314-339. https://doi.org/10.1016/j.cortex.2016.05.013

Vartiainen, J., Liljestro, M., Koskinen, M., Renvall, H., \& Salmelin, R. (2011). Functional Magnetic 
Resonance Imaging Blood Oxygenation Level-Dependent Signal and Magnetoencephalography in Reading. The Journal of Neuroscience, 31(3), 1048-1058. https://doi.org/10.1523/JNEUROSCI.3113-10.2011

Vigario, R., Sarela, J., Jousmiki, V., Hamalainen, M., \& Oja, E. (2000). Independent component approach to the analysis of EEG and MEG recordings. IEEE Transactions on Biomedical Engineering, 47(5), 589-593. https://doi.org/10.1109/10.841330

Vigneau, M., Beaucousin, V., Herv??, P. Y., Duffau, H., Crivello, F., Houd??, O., Mazoyer, B., \& TzourioMazoyer, N. (2006). Meta-analyzing left hemisphere language areas: Phonology, semantics, and sentence processing. Neurolmage. https://doi.org/10.1016/j.neuroimage.2005.11.002

Vigneau, M., Beaucousin, V., Hervé, P. Y., Jobard, G., Petit, L., Crivello, F., Mellet, E., Zago, L., Mazoyer, B., \& Tzourio-Mazoyer, N. (2010). What is right-hemisphere contribution to phonological, lexicosemantic, and sentence processing? Insights from a meta-analysis. Neurolmage, 54(1), 577-593. https://doi.org/10.1016/j.neuroimage.2010.07.036

Wang, L., Hagoort, P., \& Jensen, O. (2018). Language Prediction Is Reflected by Coupling between Frontal Gamma and Posterior Alpha Oscillations. Journal of Cognitive Neuroscience, 30(3), 432447.

Weiss, S., \& Mueller, H. M. (2012). "Too many betas do not spoil the broth": The role of beta brain oscillations in language processing. Frontiers in Psychology, 3(JUN), 1-15. https://doi.org/10.3389/fpsyg.2012.00201

Wens, V., Marty, B., Mary, A., Bourguignon, M., Op de Beeck, M., Goldman, S., Van Bogaert, P., Peigneux, P., \& De Ti??ge, X. (2015). A geometric correction scheme for spatial leakage effects in MEG/EEG seed-based functional connectivity mapping. Human Brain Mapping, 36(11), 46044621. https://doi.org/10.1002/hbm.22943

Whitney, C., Huber, W., Klann, J., Weis, S., Krach, S., \& Kircher, T. (2009). Neural correlates of narrative shifts during auditory story comprehension. Neurolmage, 47(1), 360-366. https://doi.org/10.1016/j.neuroimage.2009.04.037

Wilson, S. M., Bautista, A., Yen, M., Lauderdale, S., \& Eriksson, D. K. (2017). Validity and reliability of four language mapping paradigms. Neurolmage: Clinical, 16, 399-408. https://doi.org/10.1016/j.nicl.2016.03.015

Yarkoni, T., Poldrack, R. A., Nichols, T. E., Van Essen, D. C., \& Wager, T. D. (2011). Large-scale automated synthesis of human functional neuroimaging data. Nature Methods. https://doi.org/10.1038/nmeth. 1635

Zacà, D., Jarso, S., \& Pillai, J. J. (2013). Role of semantic paradigms for optimization of language mapping in clinical fMRI studies. American Journal of Neuroradiology, 34(10), 1966-1971. https://doi.org/10.3174/ajnr.A3628

Zaca, D., Nickerson, J. P., Deib, G., \& Pillai, J. J. (2012). Effectiveness of four different clinical fMRI paradigms for preoperative regional determination of language lateralization in patients with brain tumors. Neuroradiology, 54(9), 1015-1025. https://doi.org/10.1007/s00234-012-1056-2 